
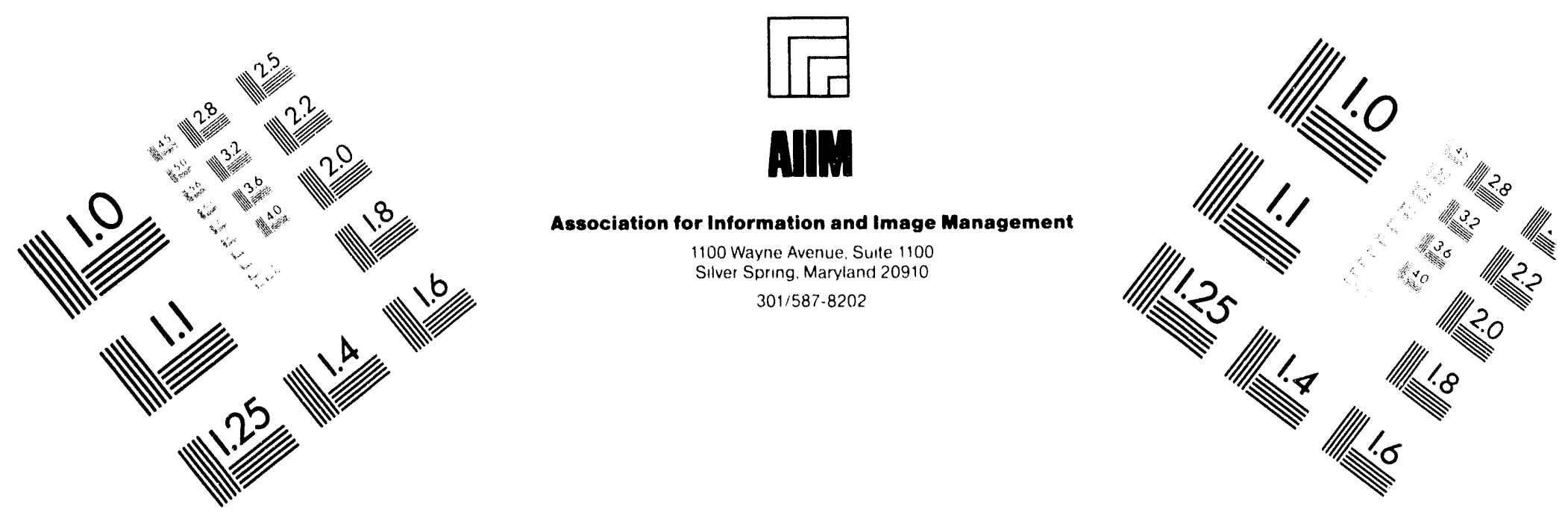

\title{
Centimeter
}

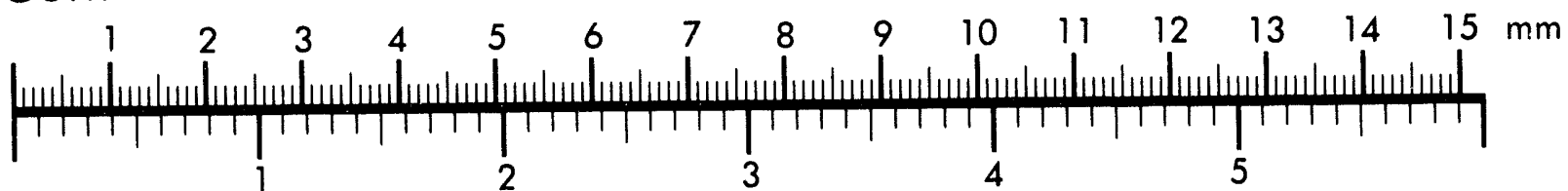
Inches
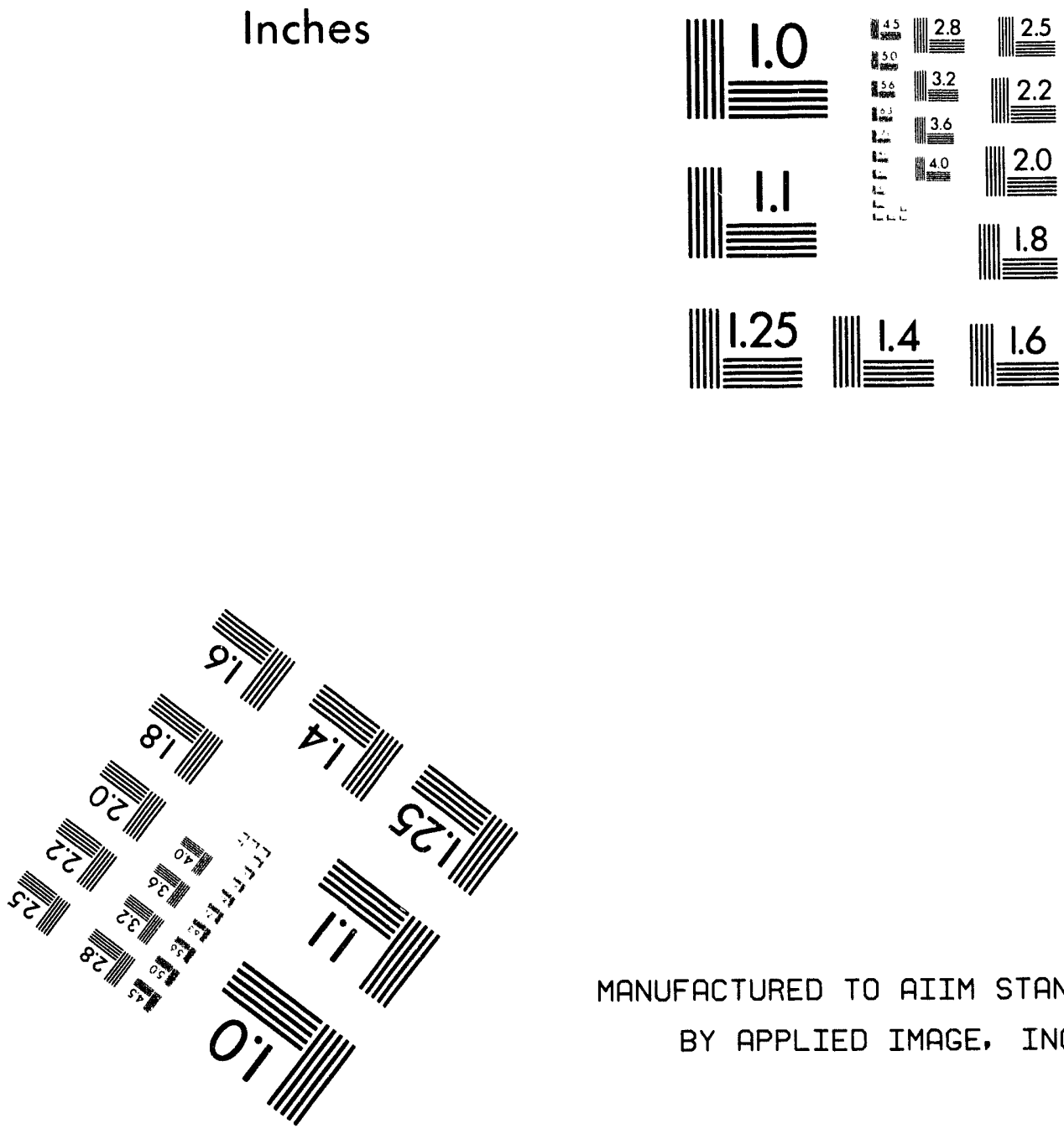

MANUFACTURED TO AIIM STANDARDS

BY APPLIED IMAGE, INC.

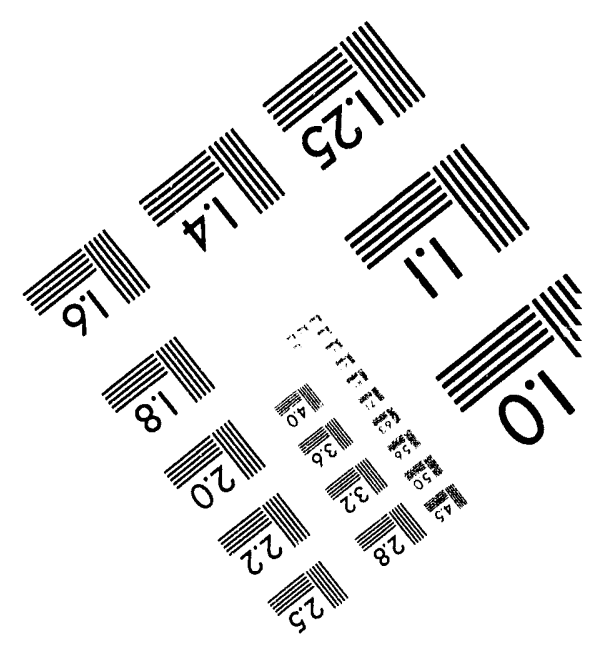



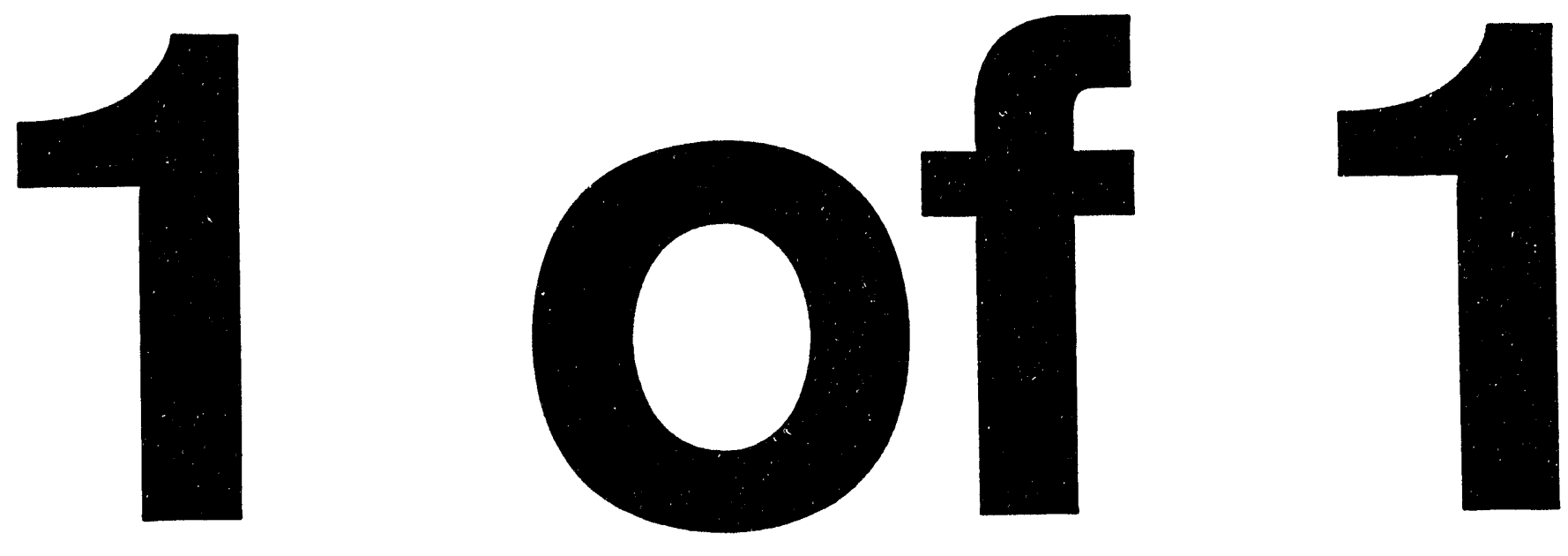


\section{POLOIDAL ROTATION AND THE EVOLUTION OF H-MODE AND VH-MODE PROFILES}

by

F.L. HINTON, G.M. STAEBLER, and Y.-B. KIM

This is a preprint of a paper presented at the Fourth H-Mode Workshop, November 15-17, 1993, Naka, Japan, and to be printed in a special issue of Plasma Physics and Controlled Fusion.

Work supported by U.S. Department of Energy

Contract No. DE-AC03-89ER51114 and

Grant No. DE-FG03-92ER54150

GENERAL ATOMICS PROJECTS 3466 and 3938 DECEMBER 1993

\section{GENERAL ATOMICS}




\title{
Poloidal rotation and the evolution of $\mathrm{H}$-mode and $\mathrm{VH}$-mode profiles
}

\author{
F I Hinton, G M Staebler and Y-B Kim \\ General Atomics, PO Box 85608, San Diego, CA 92186-9784, USA
}

\begin{abstract}
The physics which determines poloidal rotation, and its role in the development of profiles during $\mathrm{H}$ - and $\mathrm{VH}$-modes, is discussed. A simple phenomenological transport model, which incorporates the $\vec{E} \times \vec{B}$ flow shear suppression of turbulence, is shown to predict profile evolution similar to that observed experimentally during $\mathrm{H}$-mode and VH-mode.
\end{abstract}

\section{Introduction}

The suppression of turbulence which occurs at the H-transition is related to shear in the $\vec{E} \times \vec{B}$ flow (Groebner, 1993). The relation between the $\vec{E} \times \vec{B}$ flow shear and the gradients of ion density and pressure and toroidal velocity follows from the radial component of the ion momentum equation, which gives the $\vec{E} \times \vec{B}$ flow as

$$
u_{E} \equiv \frac{c E_{\mathrm{r}}}{B}=\frac{B_{\theta}}{B} u_{\phi}-\frac{B_{\phi}}{B} u_{\theta}+\frac{c}{n_{\mathrm{i}} e_{\mathrm{i}} B} \frac{\partial p_{\mathrm{i}}}{\partial r} .
$$

The effective shear parameter is actually the derivative of the Doppler shift of the unstable modes in the turbulence. As shown in Staebler et al. (1993), the derivative of $B_{\theta}$ cancels when the parallel flow contribution to the mode Doppler shift is included, so that the effective shear parameter is

$$
s_{\perp}=\frac{B_{\theta}}{B} \frac{\partial u_{\phi}}{\partial r}-\frac{c}{e B n_{i}^{2}} \frac{\partial n_{i}}{\partial r} \frac{\partial p_{i}}{\partial r},
$$

in which $\partial B / \partial r$ and $\partial^{2} p_{i} / \partial r^{2}$ have been neglected. The second derivative is neglected only for simplicity, and could affect experimental comparisons. However, the most obvious feature of the experimental profiles near the edge is their large first derivatives; the existence of nonzero second derivatives appears to be an unimportant detail. Also, the poloidal flow contribution has been neglected, for reasons which will be explained in Section 3. This makes possible a closed set of transport equations without including an additional equation for the poloidal rotation.

\section{Poloidal rotation}

The poloidal rotation is related to perpendicular and parallel flows:

$$
u_{\theta}=u_{\perp}-\frac{B_{\theta}}{B} u_{\|},
$$


where the perpendicular flow is related to the radial electric field and the pressure gradient:

$$
u_{\perp}=-\frac{c E_{\mathrm{r}}}{B}+\frac{c}{n_{\mathrm{i}} e_{\mathrm{i}} B} \frac{\partial p_{\mathrm{i}}}{\partial r}
$$

According to neoclassical theory, magnetic pumping couples the parallel and perpendicular flows, resulting in a large parallel flow, $u_{\|}=\left(B / B_{\theta}\right) u_{\perp}$ so that $u_{\theta}=0$ (neglecting a small correction due to the ion temperature gradient). Nonzero poloidal rotation results from any mechanism which reduces the parallel flow, so that its poloidal component does not cancel the perpendicular flow in Eq. (3). One such mechanism is related to orbit squeezing.

Neoclassical theory was extended (Hinton et al., 1993) to include orbit squeezing, which is the reduction of the ion banana widths due to radial electric field shear. The banana width of a barely trapped ion is

$$
\Delta r=2\left(\frac{2 \epsilon}{S}\right)^{1 / 2} \frac{v_{\perp}}{\Omega_{\theta}},
$$

where $v_{\perp}$ is its perpendicular velocity, $\Omega_{\theta}=e B_{\theta} /(m c)$, with $B_{\theta}$ the poloidal magnetic field, $\epsilon \equiv\left(B_{\max }-B_{\min }\right) /\left(2 B_{\min }\right)$, and where the squeezing factor is

$$
S=1-\frac{m c^{2}}{e B_{\theta}^{2}} \frac{\partial E_{r}}{\partial r} .
$$

It must be assumed that $\partial E_{\mathrm{r}} / \partial r<0$, consistent with the experimentally inferred electric field shear within a few centimeters of the separatrix in the DIII-D tokamak in $\mathrm{H}$-mode. The region very near, or possibly outside, the separatrix, where $E_{\mathrm{r}}$ has a zero or positive derivative, must be excluded from this analysis which does not allow for the physics which determines $E_{\mathrm{r}}$ on the open field lines.

The parallel flow is set up by the trapped ions whose density gradient gives a net parallel flow because the number of banana orbits on one side of the radius in question is different than the number on the other side. The collisional detrapping of the ions, followed by the collisional sharing of the momentum by all the untrapped ions, leads to a parallel flow given by

$$
n u_{\|}=-\frac{v_{i}^{2}}{2 S \Omega_{\theta}} \frac{\partial n}{\partial r},
$$

which is reduced by a factor $1 / S$ due to orbit squeezing. When $S>1$, the pressure gradient-driven ion parallel flow is reduced by orbit squeezing, and its poloidal component no longer cancels the diamagnetic flow, giving rise to a poloidal flow:

$$
u_{\theta}=\frac{c}{n_{\mathrm{i}} e_{\mathrm{i}} B}\left(1-\frac{1}{S}\right) \frac{\partial p_{\mathrm{i}}}{\partial r} \text {. }
$$

The poloidal flow elocity is a significant fraction of the ion diamagnetic velocity, when $S-1 \sim 1$, and is in reasonable agreement with spectroscopic measurements of the poloidal rotation velocity in helium plasmas (Kim et al., 1993) in the DIII-D tokamak in $\mathrm{H}$-mode very close to the plasma edge.

\section{Critical gradients for the $\mathrm{L}-\mathrm{H}$ transition}

It is useful to use the following simple expression for the critical flow shear (Hassam, 1991): 


$$
s_{\perp}>\left(s_{\perp}\right)_{c \mathrm{it}}=c_{h} \frac{v_{\mathrm{i}}}{R},
$$

where $c_{h}$ is a constant which will be determined, $v_{i}$ is the ion thermal velocity, and $R$ is the major radius.

We consider first the pressure gradient contribution to Eq. (2). The critical shear condition may be written as

$$
\left(\frac{c}{e B n_{\mathrm{i}}^{2}}\right) \frac{\partial n_{\mathrm{i}}}{\partial r} \frac{\partial p_{\mathrm{i}}}{\partial r}>c_{h} \frac{v_{\mathrm{i}}}{R}
$$

or

$$
L_{\mathrm{n}}<\left(\frac{\rho_{\mathrm{i}} R}{c_{h}}\right)^{1 / 2},
$$

where $\rho_{\mathrm{i}}=v_{\mathrm{i}} / \Omega_{\mathrm{i}}$, with $\Omega_{\mathrm{i}}=e_{\mathrm{i}} B / m_{\mathrm{i}}$ c. Using typical numbers for deuterium plasmas in DIII-D $\left(T_{\mathrm{i}}=400 \mathrm{eV}, B=2 \mathrm{~T}\right)$, and making the choice $c_{h}=0.2$, we find $a / L_{\mathrm{n}}>10$, which is in reasonable agreement with the density gradient length at the $\mathrm{H}$-transition.

Next, consider the toroidal velocity term. The critical shear condition is

$$
\frac{B_{\theta}}{B} \frac{u_{\phi}}{L_{\phi}}>c_{h} \frac{v_{\mathrm{i}}}{R}
$$

where $L_{\phi}$ is the gradient length for the toroidal flow velocity, $u_{\phi}$. This can be written as

$$
\frac{u_{\phi}}{v_{\mathrm{i}}} \frac{a}{L_{\phi}}>c_{k} q
$$

where $q=a B / R B_{\theta}$ is the safety factor. Using $q=5$ near the plasma edge, and the same choice $c_{h}=0.2$ as above, the right hand side is equal to 1 . Thus, if $a / L_{\phi} \simeq 2$, this requires $u_{\phi} / v_{i}>0.5$, or $u_{\phi}>70 \mathrm{~km} / \mathrm{sec}$, using helium with a temperature of $400 \mathrm{eV}$. This is, in general, too large near the plasma edge at the H-transition, so the toroidal velocity can not be important. Later in the profile evolution, when $u_{\phi}$ increases to larger values, this term starts to play an important role, and cannot be neglected at the transition to VH-mode.

Finally, consider the poloidal rotation, given by Eq. (8). The poloidal rotation becomes non-negligible only when $S-1>1$, or $\rho_{i \theta} / L_{n}>1$, or

$$
\frac{a}{L_{\mathrm{a}}}>\left(\frac{\rho_{\mathrm{i} \theta}}{a}\right)^{-1} \text {, }
$$

which is typically about 35, significantly larger than the critical value of 10 for the $\mathrm{H}$-mode transition. Thus, the poloidal rotation plays no role in suppressing the turbulent transport because $s_{\perp}$ is already large enough to suppress the L-mode turbulence, before the gradients are large enough to generate appreciable poloidal flow. It follows that poloidal rotation only occurs very near the edge, and not near the inward boundary of the good confinement zone.

\section{Transport bifurcation model}

A phenomenological transport bifurcation model was developed in Hinton and Staebler (1993), and extended in Staebler et al. (1993). The basis of this model is the nonlinear feedback implied by (i) the $\vec{E} \times \vec{B}$ flow shear suppression of turbulence 
(Biglari et al., 1991), and (ii) the simple relation between radial electric field shear and the gradients of density, temperature, and toroidal flow velocity, as given by Eq. (2). Thus, when confinement of particles, energy and toroidal angular momentum improves, the increased gradients cause a suppression of the turbulence, consistent with the confinement improvement.

The equations to be solved in determining the density, pressure, and toroidal velocity profiles are particle conservation, energy conservation, and toroidal momentum conservation:

$$
\begin{aligned}
& \frac{\partial n_{\mathrm{i}}}{\partial r}+\frac{1}{r} \frac{\partial}{\partial r} r \Gamma=S(r), \\
& \frac{3}{2} \frac{\partial p_{\mathrm{i}}}{\partial t}+\frac{1}{r} \frac{\partial}{\partial r} r Q=H(r), \\
& \frac{\partial}{\partial t}\left(m_{\mathrm{i}} n_{\mathrm{i}} u_{\phi}\right)+\frac{1}{r} \frac{\partial}{\partial r} r \Pi=M(r) .
\end{aligned}
$$

The particle, energy and momentum fluxes are expressed as functions of the density gradient, ion pressure gradient, toroidal flow velocity gradient, and the $E \times B$ flow shear as follows (Zhang and Mahajan, 1992):

$$
\begin{aligned}
& \Gamma=-\left[D_{0}+\frac{D_{1}}{1+\alpha s_{\perp}^{2}}\right] \frac{\partial n_{\mathrm{i}}}{\partial r}, \\
& Q=-\left[\chi_{0}+\frac{\chi_{1}}{1+\alpha s_{\perp}^{2}}\right] \frac{\partial p_{\mathrm{i}}}{\partial r}, \\
& \Pi=-\left[\mu_{0}+\frac{\mu_{1}}{1+\alpha s_{\perp}^{2}}\right] \frac{\partial u_{\phi}}{\partial r},
\end{aligned}
$$

where $D_{0}, D_{1}, \chi_{0}, \chi_{1}, \mu_{0}, \mu_{1}$, and $\alpha$ are the parameters of the model. The coefficients $D_{1}, \chi_{1}$ and $\mu_{1}$ are assumed to result from the part of the turbulence with long radial correlation lengths, which is suppressed by $E \times B$ flow shear, while $L_{0}, \chi_{0}$, and $\mu_{0}$ are assumed to be the remaining turbulent contributions which are not suppressed. The parameter $\alpha$ measures the strength of the $E \times B$ flow shear suppression:

$$
\alpha=\left(s_{\perp}\right)_{c r i t}^{-2} .
$$

In the following, $\alpha$ was evaluated using Eq. (9) with the edge temperature. The boundary conditions used in solving Eqs. (15) through (20) are given values for $n_{\mathrm{i}}$, $p_{i}$, and $u_{\phi}$ at $r / a=i$, and zero first derivatives for these quantities at $r-0$.

\section{Evolution of the profiles}

A code has been used (Staebler et al., 1993) to calculate the evolution of the density and temperature profiles numerically, by solving Eqs. (15) through (20). The source terms on the right hand side of these equations were taken from fits to the transport analysis of experimental data. Results for one set of parameters are shown in figure 1. An increase in the heating power initiates the $H$-transition. The high gradient layer 

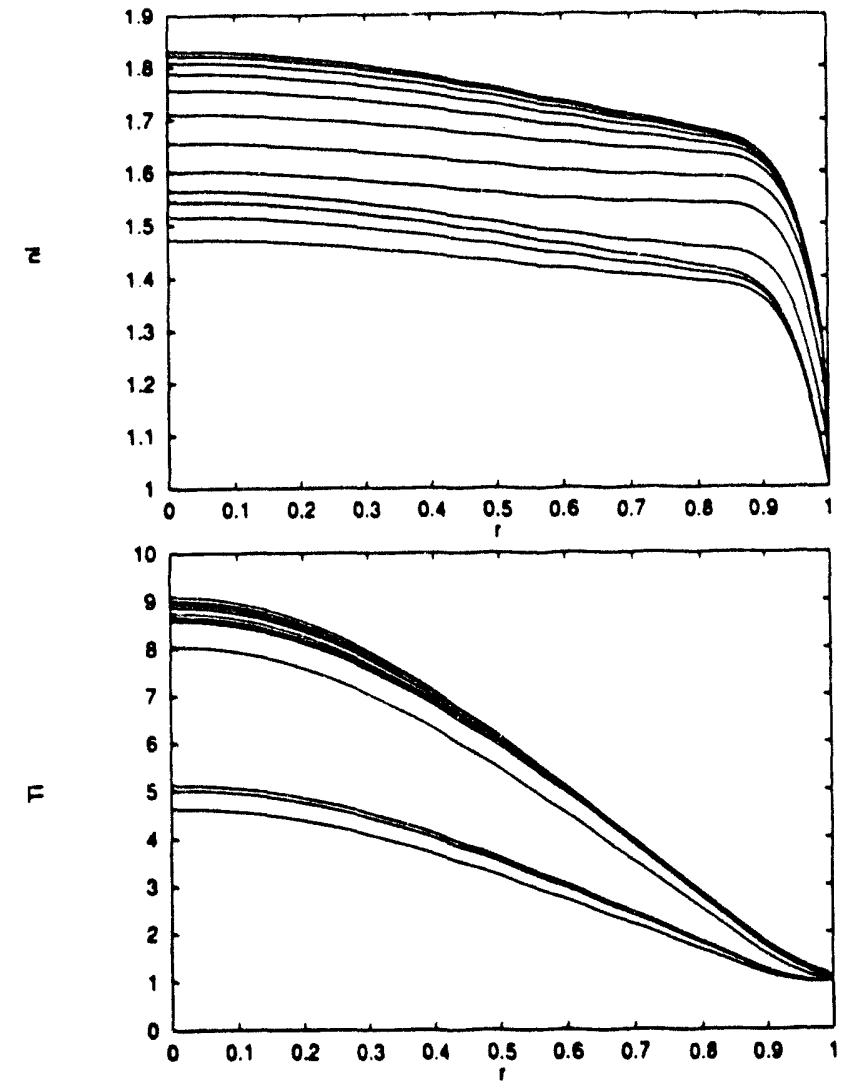

Figure 1. Density and pressure profiles for different times, showing the $\mathrm{H}$-transition.

forms first at the plasma edge. The transport barrier width increases, but quickly approaches a steady value, which is found to be relatively insensitive to the heating power.

For larger heating power, the results are shown in figure 2. The H-transition occurs very early; somewhat later, the transport barrier width increases, corresponding to the transition to VH-mode. This occurs with or without the coupling to the toroidal rotation in Eq. (2), but occurs at a lower heating power with it. This implies that VH-mode would occur with any form of heating, not just neutral beams, but that neutral beam coinjection lowers the power threshold for $\mathrm{VH}$-mode.

\section{Conclusions}

We considered the role of poloidal rotation in the development of profiles. The electric field shear which suppresses turbulence is generated by the pressure gradient and the toroidal flow, except for short times just at the transition, when it could be generated by ion orbit loss or turbulence. Poloidal rotation is determined by the physics of parallel flow, and has little or nothing to do with the subsequent development of profiles. A simple phenomenological transport model, which incorporates the $\vec{E} \times \vec{B}$ flow shear suppression of turbulence, is shown to predict profile evolution similar to that observed experimentally during $\mathrm{H}$-mode and $\mathrm{VH}$-mode. 

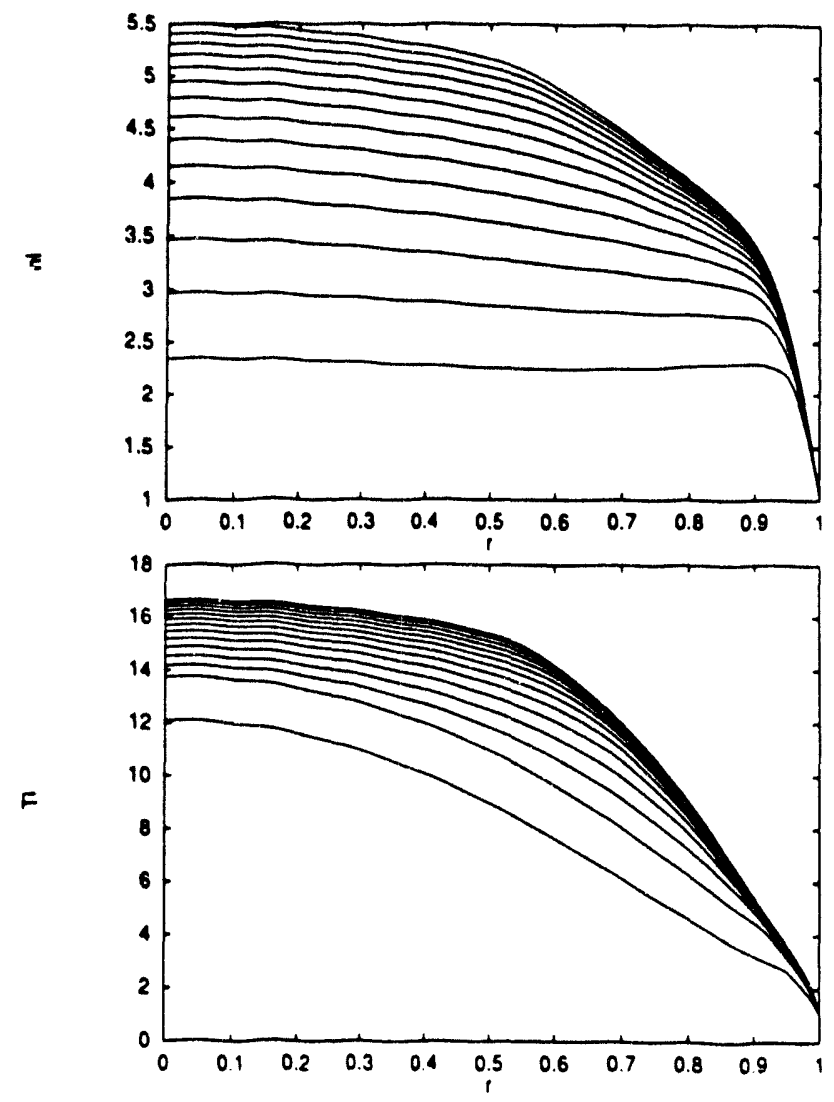

Figure 2. Density and pressure profiles for different times, showing the VH-transition.

\section{Acknowledgments}

This is a report of work sponsored by the U.S. Department of Energy under Contract No. DE-AC03-89ER51114 and Grant No. DE-FG03-92ER54150; such financial support does not constitute an endorsement by DOE of the views expressed herein.

\section{References}

Biglari H, Diamond P H and Terry P W 1991 Phys. Fluids B 21.

Groebner R J 1993 Phys. Fluids B 52343 (1993).

Hassam A B 1991 Comments Plasma Phys. and Contr. Fusion 14275.

Hinton F L and Staebler G M 1993 Phys. Fluids B 51281.

Hinton F L, Kim J, Kim Y-B, Brizard A and Burrell K H 1993 submitted to Phys. Rev. Lett.

Kim J, Burrell K H, Gohil P, Groebner R J, Kim Y-B, St John H E, Seraydarian R P and Wade M 1993 submitted to Phys. Rev. Lett.

Staebler G M, Hinton F L, Wiley J C, Dominguez R R, Greenfield C M, Gohil P, Kurki-Suonio T K and Osborne T H 1993 submitted to Phys. Fluids B.

Zhang Y Z and Mahajan S M 1992 Phys. Fluids B 41385. 

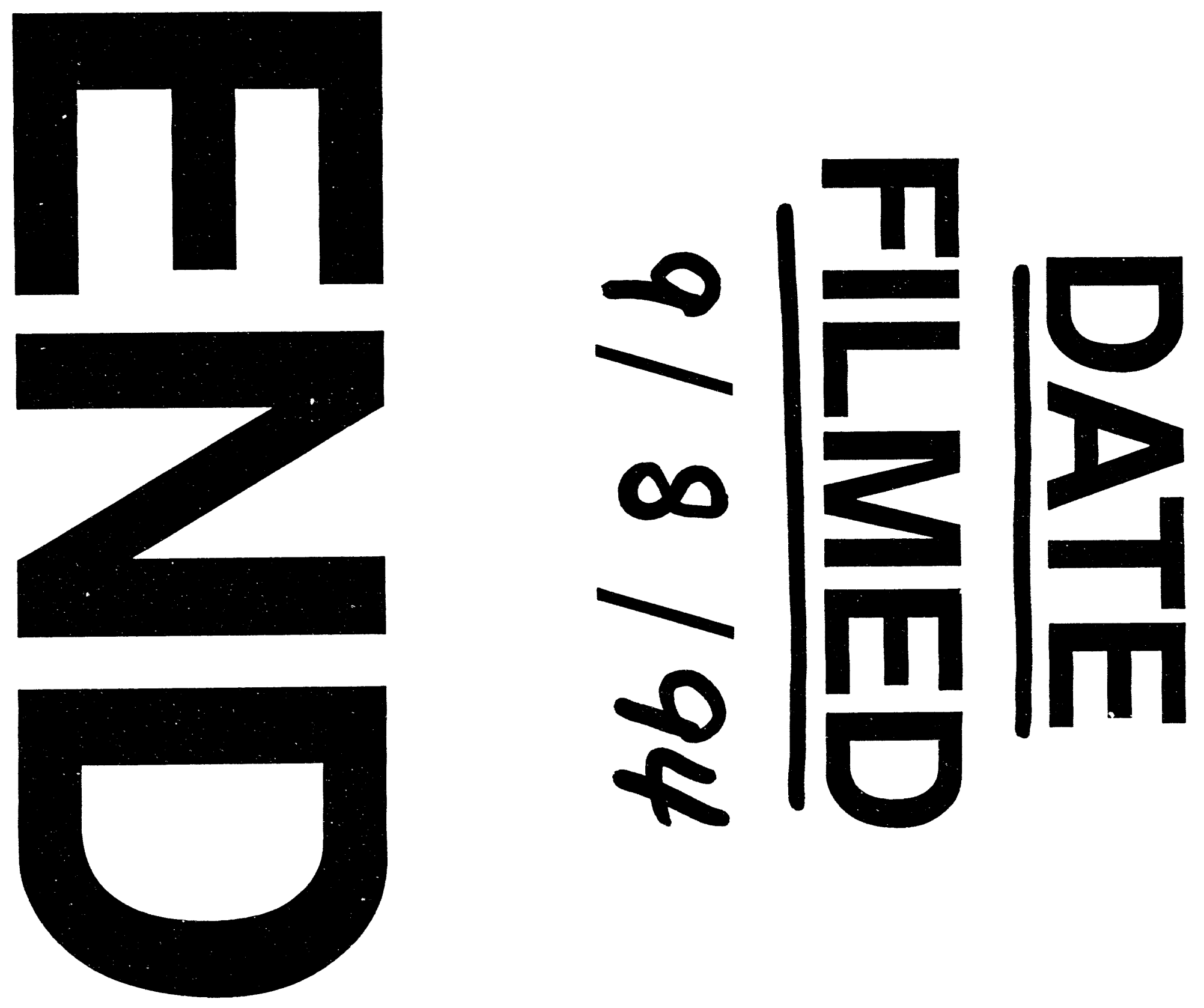
